\title{
Simulation of tension fields with in-plane rotational degrees of freedom
}

\author{
Markus Pagitz • Mostafa Abdalla
}

Received: 22 April 2010 / Accepted: 15 June 2010 / Published online: 30 June 2010

(C) The Author(s) 2010. This article is published with open access at Springerlink.com

\begin{abstract}
This paper introduces a novel multigrid approach for the geometric non-linear simulation of tension fields on the basis of a three-node membrane finite element. The element possesses, in addition to the nodal displacement degrees of freedom, an in-plane rotational degree of freedom inside the element domain that controls the direction of the tension field. This rotational degree of freedom allows the enforcement of continuity and tension field boundary conditions on the basis of a coarser mesh with varying size.
\end{abstract}

Keywords Membrane $\cdot$ Wrinkling $\cdot$ Multigrid . Finite element

\section{Introduction}

Membranes are widely used for deployable structures such as parachutes and balloons. However, despite their common use it remains a challenging task to accurately simulate their deployment. Although membranes can carry large loads in biaxial tension, they fail to carry any noteworthy compressive stresses due to the formation of wrinkles. Since the wavelength of wrinkles decreases with the membrane thickness, it is generally not possible to simulate large-scale deployable membrane structures by using shell elements. This is demonstrated in the following.

Simulation results of a sheared membrane for a decreasing thickness $t$ are shown in Fig. 1. The rectangular membrane

M. Pagitz $(\varangle) \cdot$ M. Abdalla

Aerospace Structures, Delft University of Technology,

2629 HS Delft, The Netherlands

e-mail:m.e.pagitz@tudelft.nl

M. Abdalla

e-mail: m.m.abdalla@tudelft.nl is discretized by 38,400 rotation free shell elements that are based on the Laplace-Beltrami operator and the GaussBonnet theorem, Meyer et al. [10]. It can be seen that the number of wrinkles increases from $t=1 \mathrm{~mm}$ to $t=0.1 \mathrm{~mm}$ but remains constant thereafter since the mesh is too coarse to model increasingly small wrinkles. Hence it is necessary to use a mesh density of about $10^{7}$ degrees of freedom (dof) per square meter to accurately simulate a membrane with a thickness between $t=0.01-0.1 \mathrm{~mm}$. For example, currently used scientific balloons with a diameter of $80 \mathrm{~m}$ are made from a $0.04 \mathrm{~mm}$ thick membrane and possess a surface area of $16,000 \mathrm{~m}^{2}$, Pagitz and Pellegrino [15]. The resulting model would consists of approximately $10^{11}$ dof and thus can not be processed by existing computers in a reasonable amount of time.

Since the bending energy is generally small compared to the total strain energy of a membrane, it is possible to overcome this computational limitation by using an artificial bending stiffness that prevents mesh dependent buckling patterns and a modified material law or deformation gradient that removes the remaining compressive stresses. The drawback of this approach is that information about the number of wrinkles and their amplitudes is lost. However, the loss of some detail information is an acceptable price for reducing the number of dof by several orders of magnitude. Although existing thin-shell finite elements are technically mature, the development of techniques that eliminate compressive membrane stresses is still an active field of research, Jenkins [4].

\section{Literature review}

Tension field theory, Steigmann [22], provides the theoretical framework for methods that eliminate compressive stresses in membranes. The first contribution to tension field theory 

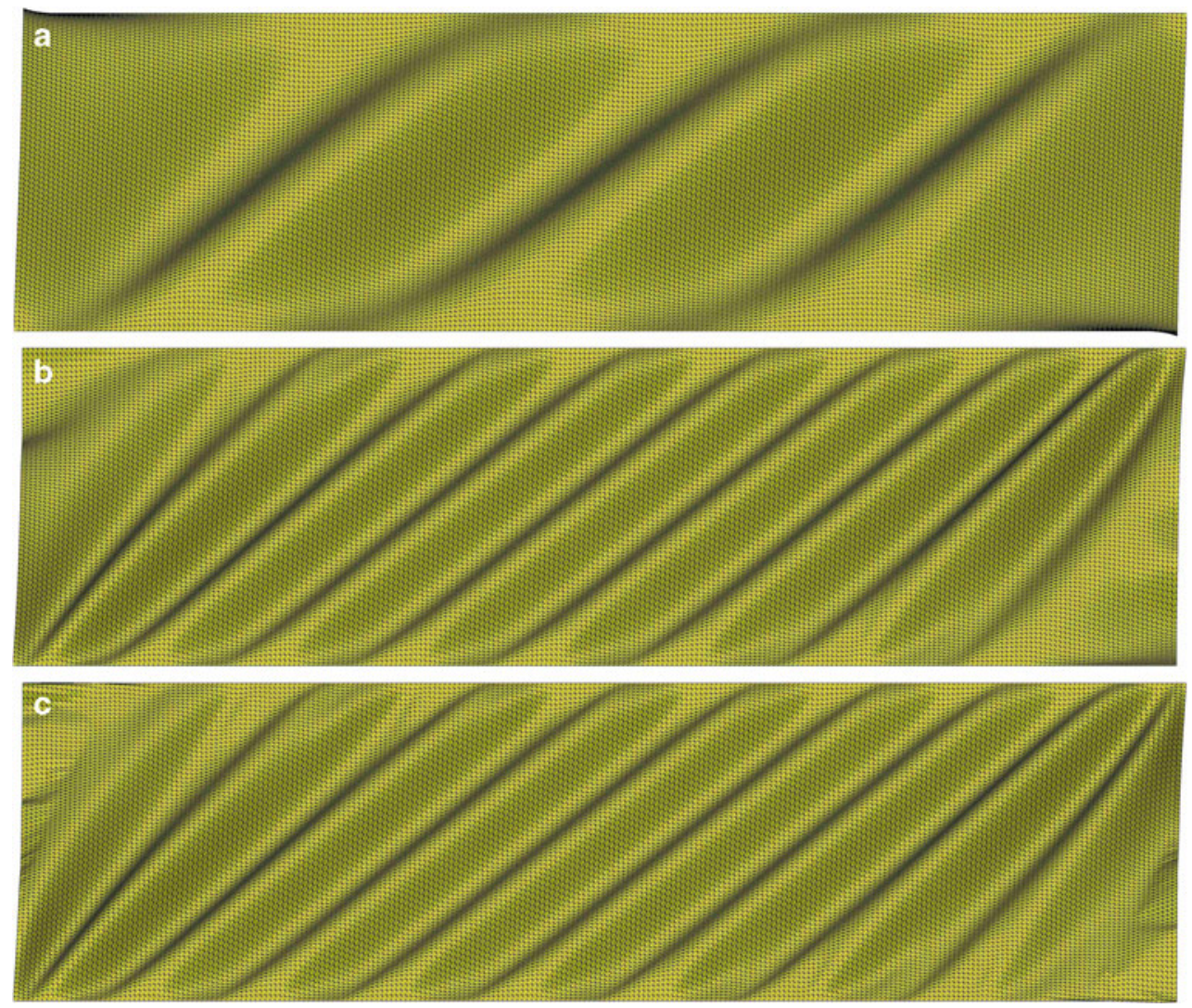

Fig. 1 Simulation results of a sheared rectangular membrane $(375 \mathrm{~mm} \times 125 \mathrm{~mm} \times t)$. Lower edge is fixed and upper edge is moved horizontally by $\Delta u=3 \mathrm{~mm}$ : a $t=1 \mathrm{~mm}, \mathbf{b} t=0.1 \mathrm{~mm}, \mathbf{c} t=0.01 \mathrm{~mm}$

was by Wagner [25] in 1929. He investigated the behavior of thin metal webs in beams that carry a shear load far beyond the initial buckling value. Wagner concluded that compressive stresses in these webs are negligible once the initial buckling value is reached and that increasingly large loads are purely carried by a tension field.

There exists a small number of analytical solutions for wrinkled membranes. Equations for an initially unstressed, annular membrane under torsional loading were first derived by Reissner [17]. The assumption of an initially unstressed membrane simplifies the derivation since the whole membrane domain is wrinkled so that the direction of the tension field is the only unknown. Stein and Hedgepeth [23] extended the work of Reissner by considering an initially stressed membrane of infinite size. A consequence of the initial prestress and the infinite dimension is that the membrane is only partially wrinkled. The wrinkled region was modeled by using a varying Poisson's ratio such that the compressive stresses vanish. The work of Stein and Hedgepeth was generalized by Mikulas [11] for prestressed membranes of finite size. Li and Steigmann [6] finally derived equations for finite rotations of the central hub. An analytical solution for a completely wrinkled rectangular membrane, subject to various loading conditions, was published by Mansfield [9].
He approached the problem by maximizing the strain energy of the tension field with the help of variational calculus. ${ }^{1} \mathrm{~A}$ nice property of Mansfield's solution is that the direction of the tension field varies over the membrane domain. Furthermore, for pure shear, both the membrane stresses and the gradient of the tension field have singularities which makes it a challenging benchmark test. We will use the rectangular membrane in the following to demonstrate the good performance of the proposed method since the simulation of an annular membrane under torsional loading is trivial.

The availability of increasingly powerful computers in the 1980s sparked the development of algorithms for the simulation of wrinkled membranes. These algorithms can generally be grouped into two categories:

The first category eliminates compressive stresses by modifying the material law. For example, Miller [12] developed an algorithm that uses, like the analytical work by Stein and Hedgepeth [23], a variable Poisson's ratio. An implementation of this algorithm into commercial finite element

\footnotetext{
1 Although Mansfield's solution is based on an isotropic membrane, it can be extended to orthotropic materials since a Young's modulus that is a function of the wrinkle direction does not alter the nature of the underlying functional.
} 
packages was undertaken by Adler [1]. Liu et al. [7] introduced a penalty parameter to reduce the compressive stresses by softening the stiffness of the membrane orthogonal to the wrinkle direction. A similar method that is based on a pseudodynamic solution strategy was subsequently published by Rossi et al. [20]. Akita et al. [2] used a projection method to extract the elastic part from the total strains of a wrinkled finite element. A related algorithm for isotropic and orthotropic membranes was recently published by Jarasjarungkiat et al. [3].

Unlike the first category, the second group of algorithms eliminates compressive stresses by modifying the deformation gradient. For example, Wu and Canfield [26] developed a method that modifies the deformation gradient for the simulation of isotropic membranes. A generalized version of this algorithm for isotropic and anisotropic membranes was subsequently published by Roddeman et al. [18,19]. Muttin [14] derived a quadrilateral finite element that is based on Roddeman's work and Lu et al. [8] extended Muttin's work by deriving explicit equations for the force vector and tangent stiffness matrix. Further publications that emerged from the paper by Wu and Canfield are, for example, Kang and Im [5], Schoop et al. [21], Raible et al. [16] and Mosler [13].

This paper introduces a novel multigrid approach for the geometric non-linear simulation of tension fields on the basis of a three-node membrane finite element. The element possesses, in addition to the nodal displacement degrees of freedom, an in-plane rotational degree of freedom inside the element domain that controls the direction of the tension field. This rotational degree of freedom allows the enforcement of continuity and tension field boundary conditions on the basis of a coarser mesh with varying size. The outline of the remaining paper is as follows: Sect. 3 presents the derivation of the finite element and Sect. 4 shows that alternative equilibria are a source for the well known convergence problems that can occur during the simulation of wrinkled membranes. Section 5 introduces a multigrid approach that is used to avoid local optima in wrinkled membranes and Sect. 6 provides numerical examples that demonstrate the good performance of the proposed method. Section 7 concludes the paper.

\section{Derivation of finite element}

This section presents the derivation of a three-node membrane finite element with an in-plane rotational dof inside the element domain that controls the direction of the tension field. The chosen node numbering and local coordinate system is identical to the standard triangular membrane finite element by Tabarrok and Qin [24]. The direction of the tension field is defined by a unit vector that emanates from the origin of the coordinate system and encloses an angle $\alpha$ with the $x$-axis, Fig. 2.

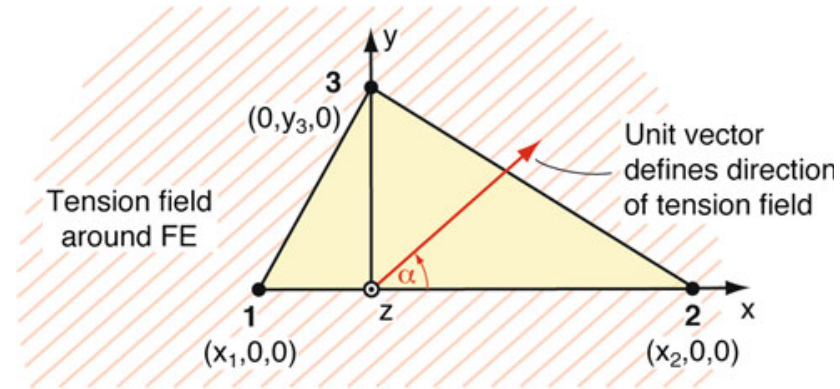

Fig. 2 Local coordinate system and wrinkle direction of finite element
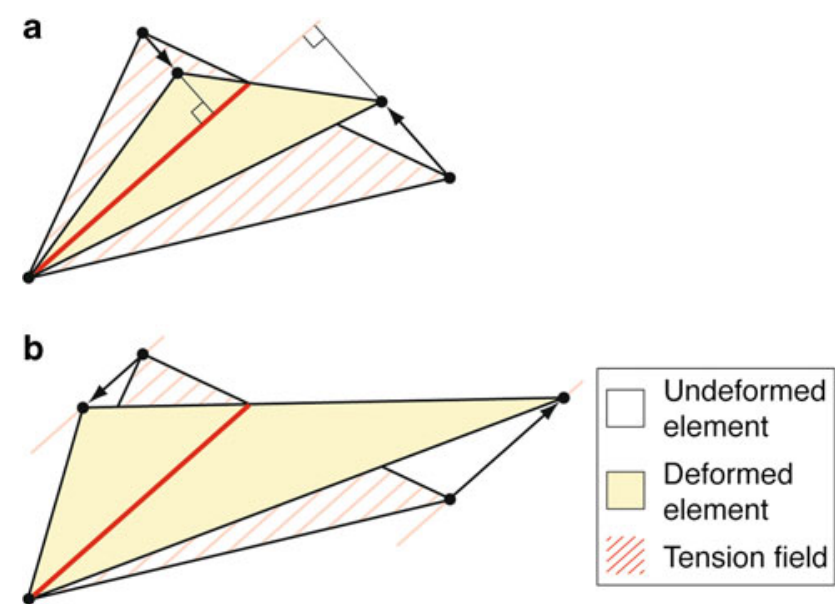

Fig. 3 Additional zero energy modes of finite element: a Wrinkling zero energy mode; $\mathbf{b}$ Shear zero energy mode

The displacements $\left[u_{i} v_{i} w_{i}\right]$ of node $i$ can be linearly interpolated over the element domain by

$$
\begin{aligned}
{\left[\begin{array}{lll}
u & v & w
\end{array}\right]=} & (\mathbf{a}+\mathbf{b} x+\mathbf{c} y)^{\mathrm{T}}[\mathbf{u} \mathbf{v} \mathbf{w}] \\
= & \frac{1}{2 A}\left(\left[\begin{array}{c}
x_{2} y_{3} \\
-x_{1} y_{3} \\
0
\end{array}\right]+\left[\begin{array}{c}
-y_{3} \\
y_{3} \\
0
\end{array}\right] x\right. \\
& \left.+\left[\begin{array}{c}
-x_{2} \\
x_{1} \\
x_{2}-x_{1}
\end{array}\right] y\right)^{\mathrm{T}}\left[\begin{array}{lll}
u_{1} & v_{1} & w_{1} \\
u_{2} & v_{2} & w_{2} \\
u_{3} & v_{3} & w_{3}
\end{array}\right]
\end{aligned}
$$

where $A=\left(x_{2}-x_{1}\right) y_{3} / 2$ is the element area. A consequence of the linear shape functions is that the element can only model constant stress and strain states so that the resulting tension field is unidirectional. The Green-Lagrange strain of a tension ray within the displacement field of the finite element is

$\varepsilon=\frac{L^{2}-1}{2}$ 
Fig. 4 Sheared and stretched rectangular membrane with indicated wrinkles

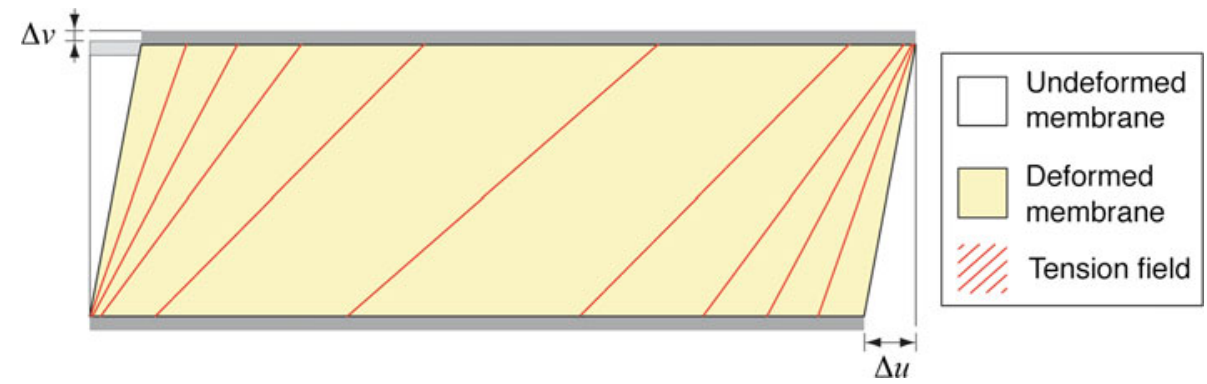

The undeformed tension ray has a unit length and the deformed length $L$ results in

$L=|\mathbf{n}|=\left|\begin{array}{c}\cos (\alpha)+\mathbf{p}^{\mathrm{T}} \mathbf{u} \\ \sin (\alpha)+\mathbf{p}^{\mathrm{T}} \mathbf{v} \\ \mathbf{p}^{\mathrm{T}} \mathbf{w}\end{array}\right|$

where $\mathbf{p}=\left[\begin{array}{l}p_{1} \\ p_{2} \\ p_{3}\end{array}\right]=\mathbf{b} \cos (\alpha)+\mathbf{c} \sin (\alpha)$

and therefore

$$
\begin{aligned}
\varepsilon= & \frac{1}{2}\left(\left(\mathbf{p}^{\mathrm{T}} \mathbf{u}\right)^{2}+\left(\mathbf{p}^{\mathrm{T}} \mathbf{v}\right)^{2}+\left(\mathbf{p}^{\mathrm{T}} \mathbf{w}\right)^{2}\right) \\
& +\cos (\alpha)\left(\mathbf{p}^{\mathrm{T}} \mathbf{u}\right)+\sin (\alpha)\left(\mathbf{p}^{\mathrm{T}} \mathbf{v}\right) .
\end{aligned}
$$

It is important to note that the strain of a tension ray is not only a function of the nodal displacements but also of the angle $\alpha$ between the tension field and the $x$-axis of the finite element. The corresponding rotational dof is not fixed to a certain location within the finite element since $\alpha$ can be measured from any tension ray.

The potential energy $\Pi$ of the finite element is

$\Pi\left(\mathbf{u}^{\star}, \alpha\right)=\frac{1}{2} E \operatorname{At\varepsilon }\left(\mathbf{u}^{\star}, \alpha\right)^{2}$

where $E$ is the Young's modulus and $t$ the thickness of the membrane. The force vector $\mathbf{f}$ is

$$
\begin{aligned}
\mathbf{f} & =\left[\begin{array}{l}
\partial \Pi / \partial \mathbf{u}^{\star} \\
\partial \Pi / \partial \alpha
\end{array}\right]=E A t \varepsilon\left[\begin{array}{l}
\partial \varepsilon / \partial \mathbf{u}^{\star} \\
\partial \varepsilon / \partial \alpha
\end{array}\right] \\
& =E A t \varepsilon\left[\begin{array}{l}
p_{1} \mathbf{n} \\
p_{2} \mathbf{n} \\
p_{3} \mathbf{n} \\
\mathbf{n}^{\prime T} \mathbf{n}
\end{array}\right]=E A t \varepsilon \mathbf{r}
\end{aligned}
$$

where

$\mathbf{u}^{\star}=\left[\begin{array}{lllllllll}u_{1} & v_{1} & w_{1} & u_{2} & v_{2} & w_{2} & u_{3} & v_{3} & w_{3}\end{array}\right]^{\mathrm{T}}$

and $\mathbf{n}^{\prime}=\partial \mathbf{n} / \partial \alpha$. The force vector does not only contain force terms but additionally a moment that is energetically conjugate to $\alpha$. Although this moment is coupled to the nodal forces, it originates from within the finite element domain. Hence it does not have to be transformed for the element assembly nor is it merged with any other dof. Finally, the material stiffness matrix is

$\mathbf{K}_{m}=E A t \mathbf{r} \mathbf{r}^{\mathrm{T}}$

and the geometric stiffness matrix results in

$\mathbf{K}_{g}=$ Eate $\left[\begin{array}{ccc|ccc|ccc|c}p_{1}^{2} & 0 & 0 & p_{1} p_{2} & 0 & 0 & p_{1} p_{3} & 0 & 0 & p_{1} n_{1}^{\prime}+p_{1}^{\prime} n_{1} \\ & p_{1}^{2} & 0 & 0 & p_{1} p_{2} & 0 & 0 & p_{1} p_{3} & 0 & p_{1} n_{2}^{\prime}+p_{1}^{\prime} n_{2} \\ & & p_{1}^{2} & 0 & 0 & p_{1} p_{2} & 0 & 0 & p_{1} p_{3} & p_{1} n_{3}^{\prime}+p_{1}^{\prime} n_{3} \\ \hline & & & p_{2}^{2} & 0 & 0 & p_{2} p_{3} & 0 & 0 & p_{2} n_{1}^{\prime}+p_{2}^{\prime} n_{1} \\ & & & & p_{2}^{2} & 0 & 0 & p_{2} p_{3} & 0 & p_{2} n_{2}^{\prime}+p_{2}^{\prime} n_{2} \\ & & & & p_{2}^{2} & 0 & 0 & p_{2} p_{3} & p_{2} n_{3}^{\prime}+p_{2}^{\prime} n_{3} \\ \hline & & & & & p_{3}^{2} & 0 & 0 & p_{3} n_{1}^{\prime}+p_{3}^{\prime} n_{1} \\ & & & & & & p_{3}^{2} & 0 & p_{3} n_{2}^{\prime}+p_{3}^{\prime} n_{2} \\ & & & & & & & p_{3}^{2} & p_{3} n_{3}^{\prime}+p_{3}^{\prime} n_{3} \\ \hline \text { Sym. } & & & & & & & \mathbf{n}^{\prime \mathrm{T}} \mathbf{n}^{\prime}-\mathbf{n}^{\mathrm{T}} \mathbf{n}\end{array}\right]$

where $\mathbf{p}^{\prime}=\partial \mathbf{p} / \partial \alpha$. The corresponding equations for an anisotropic membrane can be obtained by replacing $E$ with $E(\alpha)$ in Eq. 5.

The finite element possesses nine nodal dof. Six dof can be associated with rigid body displacements and rotations. Another two modes are, in contrast to a standard membrane finite element, additional zero energy modes. They satisfy $\Delta L=0$ such that

$\mathbf{p}^{\mathrm{T}} \mathbf{u}=0$ and $\mathbf{p}^{\mathrm{T}} \mathbf{v}=0$

which results in a coupling of the in-plane nodal displacements

$u_{1}=a u_{2}+b u_{3} \quad$ and $\quad v_{1}=a v_{2}+b v_{3}$

where

$a=\frac{y_{3}+x_{1} \tan (\alpha)}{y_{3}+x_{2} \tan (\alpha)}$ and $b=\frac{\left(x_{2}-x_{1}\right) \tan (\alpha)}{y_{3}+x_{2} \tan (\alpha)}$.

\begin{tabular}{|c|c|c|c|c|c|c|}
\hline \multicolumn{3}{|c|}{ Geometry } & \multicolumn{2}{|c|}{ Material } & \multicolumn{2}{|c|}{ Displacements } \\
\hline Width & Height & $t$ & $E$ & $v$ & $\Delta u$ & $\Delta v$ \\
\hline 375 & 125 & 0.01 & 3,500 & 0 & 3 & {$[1,2,3]$} \\
\hline
\end{tabular}

Table 1 Geometric, material and loading parameters of sheared and stretched rectangular membrane (units are $\mathrm{N}$ and $\mathrm{mm}$ ) 
Fig. 5 Comparison of analytical and numerical wrinkle directions for $\Delta u=3 \mathrm{~mm}$ and different values of $\Delta v$ : a Triangular mesh (2882 elements); b $\Delta v=1 \mathrm{~mm}$; c $\Delta v=2 \mathrm{~mm} ; \mathbf{d} \Delta v=3 \mathrm{~mm}$ a

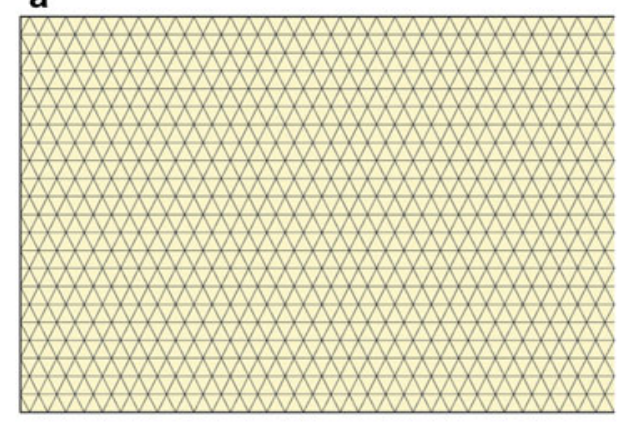

C

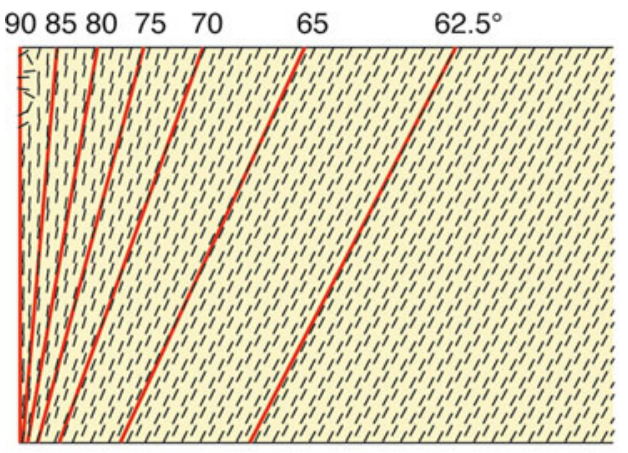

b

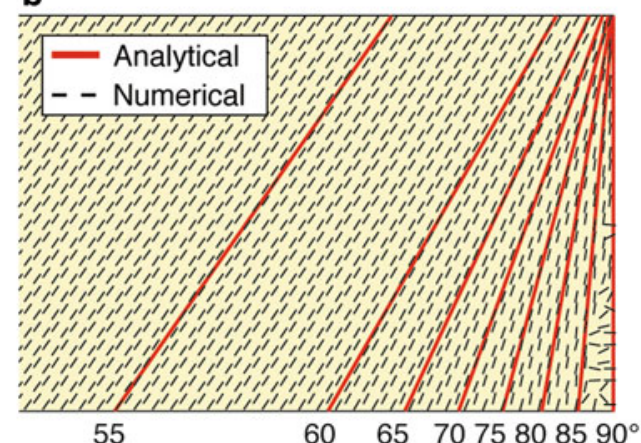

55

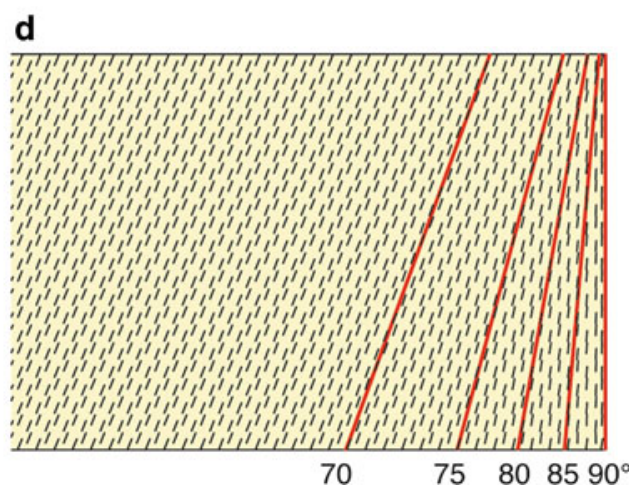

Figure 3 shows the two additional zero energy modes. The first mode eliminates stresses orthogonal to the wrinkle direction and the second mode eliminates shear stresses.

\section{Local optima in wrinkled membranes}

A rectangular membrane where the lower edge is fixed and the upper edge is moved parallel to the lower edge is used in the following as a benchmark test, Fig. 4. Note that the complete membrane is wrinkled, independent of the vertical displacement $\Delta v$ for any non-zero horizontal displacement $\Delta u$, Mansfield [9]. The considered geometric, material and loading parameters are summarized in Table 1.

The simulations were carried out with a purpose made finite element program that utilizes the Newton-Raphson method. The maximum change of a single wrinkle direction during every iteration was limited to $\alpha_{\max }=0.4 \mathrm{rad}$. The initial tension field for the simulations is based on the directions of the first principal stresses of an unwrinkled, isotropic membrane. Note that $v$ was chosen arbitrarily and does not need to be zero.

Analytical and numerical wrinkle directions of the sheared and stretched rectangular membrane are shown in Fig. 5. It can be seen that the simulation results are in good agreement with the analytical solution. Nevertheless, there are small regions at the top-left and bottom-right corners that increase for decreasing values of $\Delta v$ where the converged wrinkle directions appear to be random. Figure 6 shows the corresponding convergence plot of the residual nodal force vector (contains only nodal forces, no moments). Although it takes only 11 iterations for $\Delta v=3 \mathrm{~mm}$ it needs more than 120 iterations for $\Delta v=1 \mathrm{~mm}$ due to the increasingly large regions of disordered wrinkles. The physics of this phenomenon is discussed in the following.

Figure 7 shows a square membrane that is discretized by two finite elements and subjected to two different prescribed displacements. Furthermore, the strain, moment and rotational stiffness of the first element are shown as a function of the wrinkle direction $\alpha$ together with the resulting equilibrium configurations for the dyed regions. An important result is that the range of initial wrinkle directions for which a simulation converges to the desired equilibrium configuration decreases for increasingly wrinkled and decreasingly stretched membranes. Furthermore, besides the desired equilibrium configuration, there exist up to three undesired equilibria to which a numerical simulation can converge. Since standard solution strategies are not capable of "jumping" from one convex region with a local optima to another region with a global optima, it is obvious that the final result depends on the initial wrinkle directions.

As the top-left and bottom-right corners of a sheared membrane possess only small tensile and comparatively large compressive stresses, it is not surprising that these regions 
Fig. 6 Convergence of residual nodal force vector $\mathbf{f}_{r}$ for isotropic and wrinkled rectangular membrane
Fig. 7 a Square membrane subjected to two different prescribed displacements; b Strain, moment and rotational stiffness of first element as a function of $\alpha$ $\left(E=3,500 \mathrm{~N} / \mathrm{mm}^{2}\right.$ and $t=1 \mathrm{~mm})$

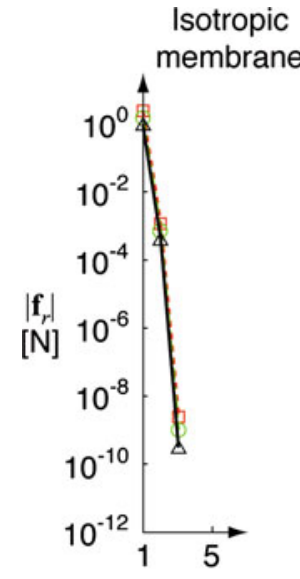

Wrinkled membrane

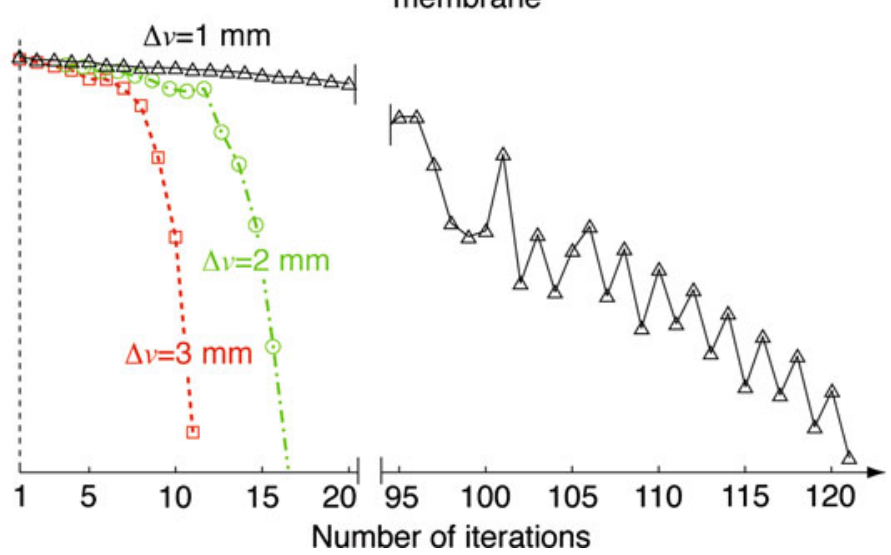

a

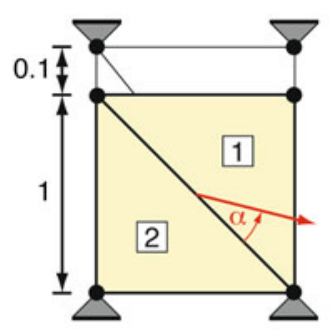

b
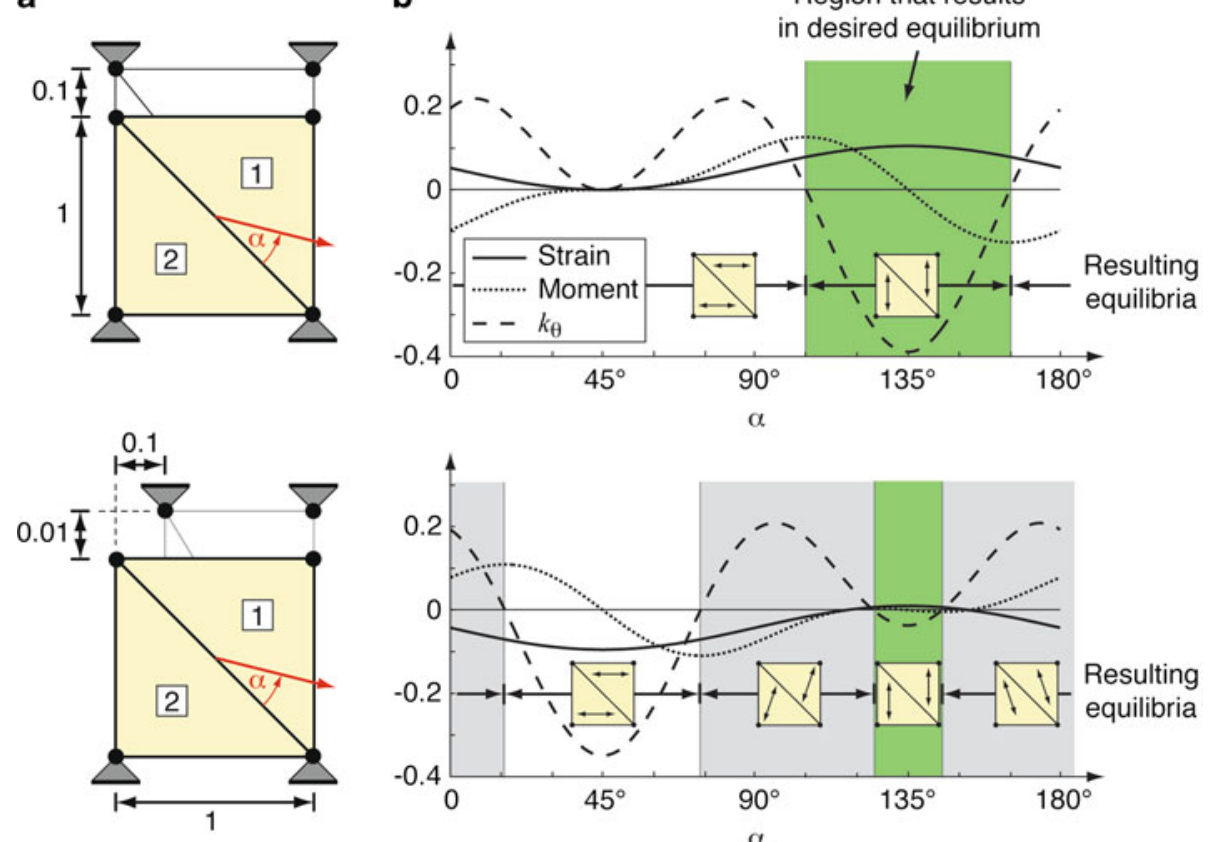

converge towards alternative equilibria. It is shown in the next section how local optima can be avoided by using a multigrid approach.

\section{Multigrid for rotations and displacements}

It was shown in the last section that there are regions at the top-left and bottom-right corners of the sheared and stretched rectangular membrane where the converged wrinkle directions appear to be random. Furthermore it was shown that their size has a severe impact on the convergence rate. In the following we present a multigrid approach that overcomes the previously observed problems.

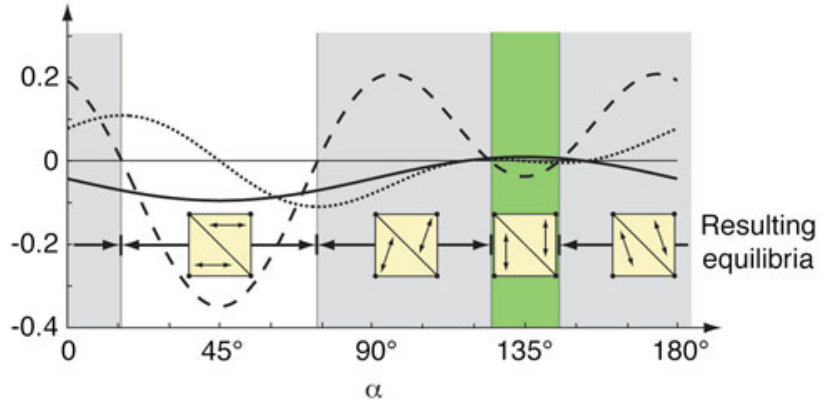

The directions of the first principal stresses from an unwrinkled isotropic membrane are interpolated with a second, coarser grid of quadrilateral elements by using the method of least squares. The triangular and quadrilateral grid are illustrated in Fig. 8. The interpolation matrix $\mathbf{N}$ contains the values of the quadrilateral shape functions at the centre of gravities $\left(x_{c}, y_{c}\right)$ of the triangular elements. The size of $\mathbf{N}$ is $e_{t} \times n_{q}$ where $e_{t}$ is the number of triangles and $n_{q}$ the number of nodes of the quadrilateral grid.

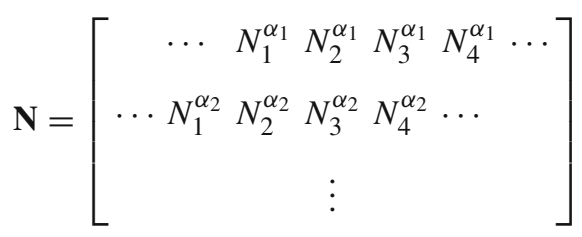


Fig. 8 a Triangular mesh with displacement dof at nodes and rotational dof inside element domains; b Quadrilateral mesh with rotational dof at nodes; c Multigrid, element rotations are interpolated from quadrilaterals

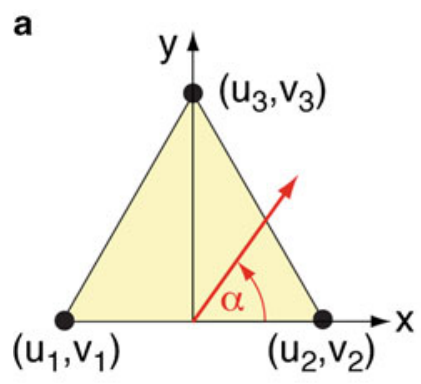

b
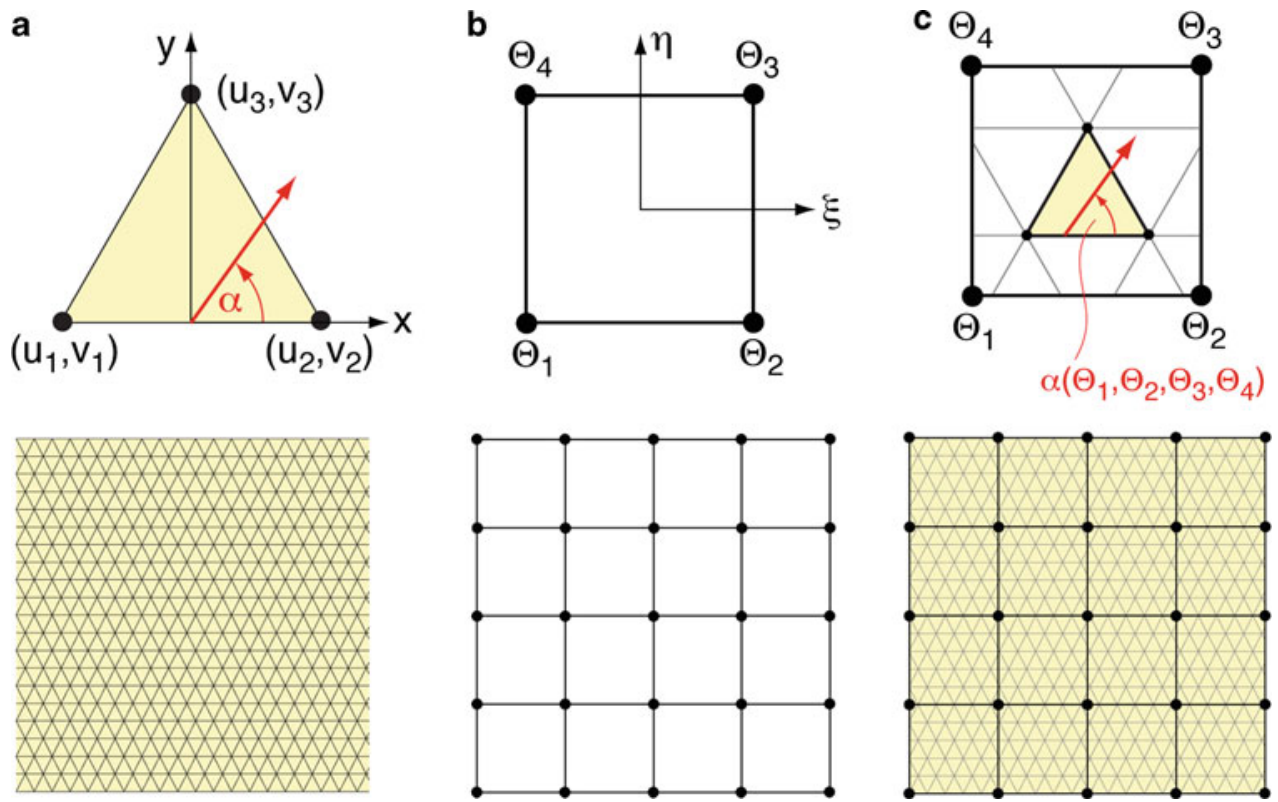

Fig. 9 a Least square interpolation of first principal stresses $\sigma_{1}$ from an unwrinkled isotropic membrane with three quadrilaterals; b Enforced wrinkle boundary conditions (BC)
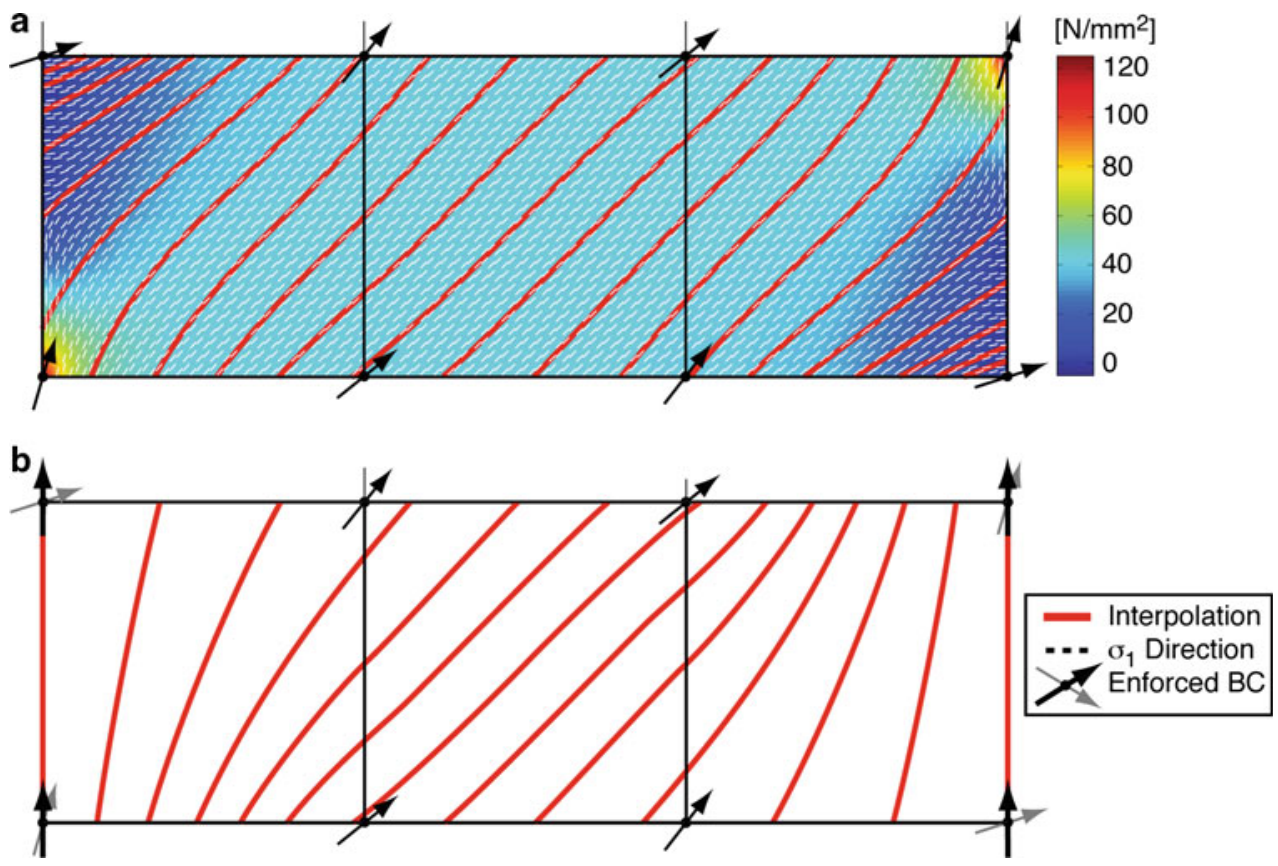

The quadrilateral shape functions are

$$
\begin{aligned}
& N_{1}^{\alpha_{i}}=\frac{1}{4}\left(1-\xi^{\alpha_{i}}\right)\left(1-\eta^{\alpha_{i}}\right) ; N_{2}^{\alpha_{i}}=\frac{1}{4}\left(1+\xi^{\alpha_{i}}\right)\left(1-\eta^{\alpha_{i}}\right) \\
& N_{3}^{\alpha_{i}}=\frac{1}{4}\left(1+\xi^{\alpha_{i}}\right)\left(1+\eta^{\alpha_{i}}\right) ; N_{4}^{\alpha_{i}}=\frac{1}{4}\left(1-\xi^{\alpha_{i}}\right)\left(1+\eta^{\alpha_{i}}\right)
\end{aligned}
$$

where $\xi^{\alpha_{i}}, \eta^{\alpha_{i}} \in[-1,1]$ are the local coordinates at the centre of gravity of the $i$ th triangle. The values of $\xi$ and $\eta$ for an orthogonal mesh are

$$
\begin{aligned}
& \xi=\frac{4 x_{c}-\left(x_{1}+x_{2}+x_{3}+x_{4}\right)}{-x_{1}+x_{2}+x_{3}-x_{4}} \\
& \eta=\frac{-4 y_{c}+\left(y_{1}+y_{2}+y_{3}+y_{4}\right)}{y_{1}+y_{2}-y_{3}-y_{4}}
\end{aligned}
$$

where $\left(x_{j}, y_{j}\right)$ are the nodal coordinates of the quadrilateral that encloses the triangle. The vector $\mathbf{t}$ of size $n_{q}$ contains the unknown rotations $\Theta$ at the nodes of the quadrilateral grid

$$
\mathbf{t}=\left[\Theta_{1} \Theta_{2} \Theta_{3} \cdots\right]^{\mathrm{T}}
$$


Discretizations

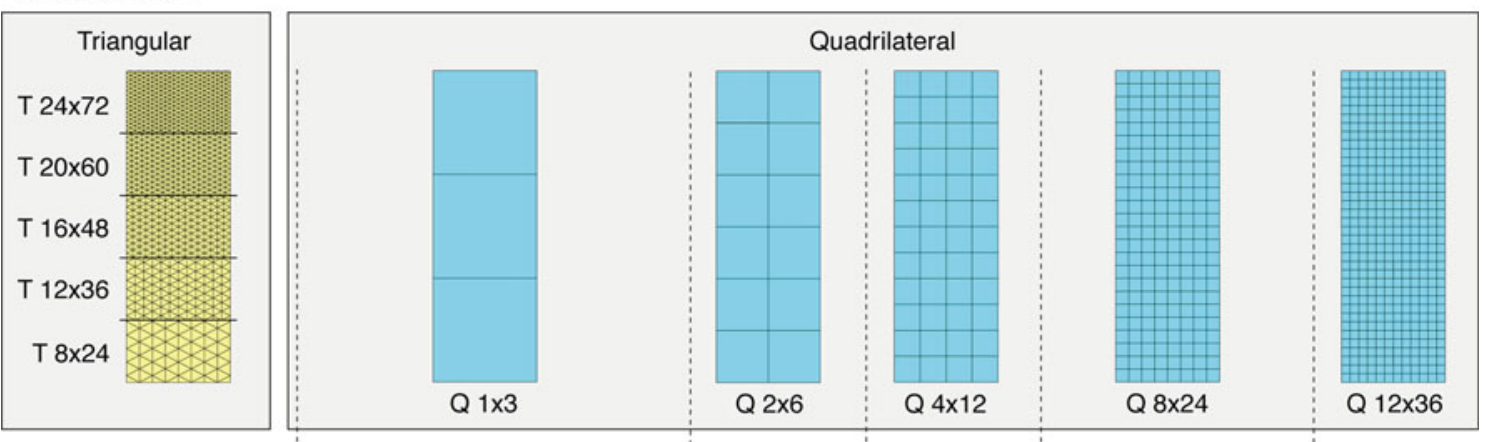

Convergence of residual force vector

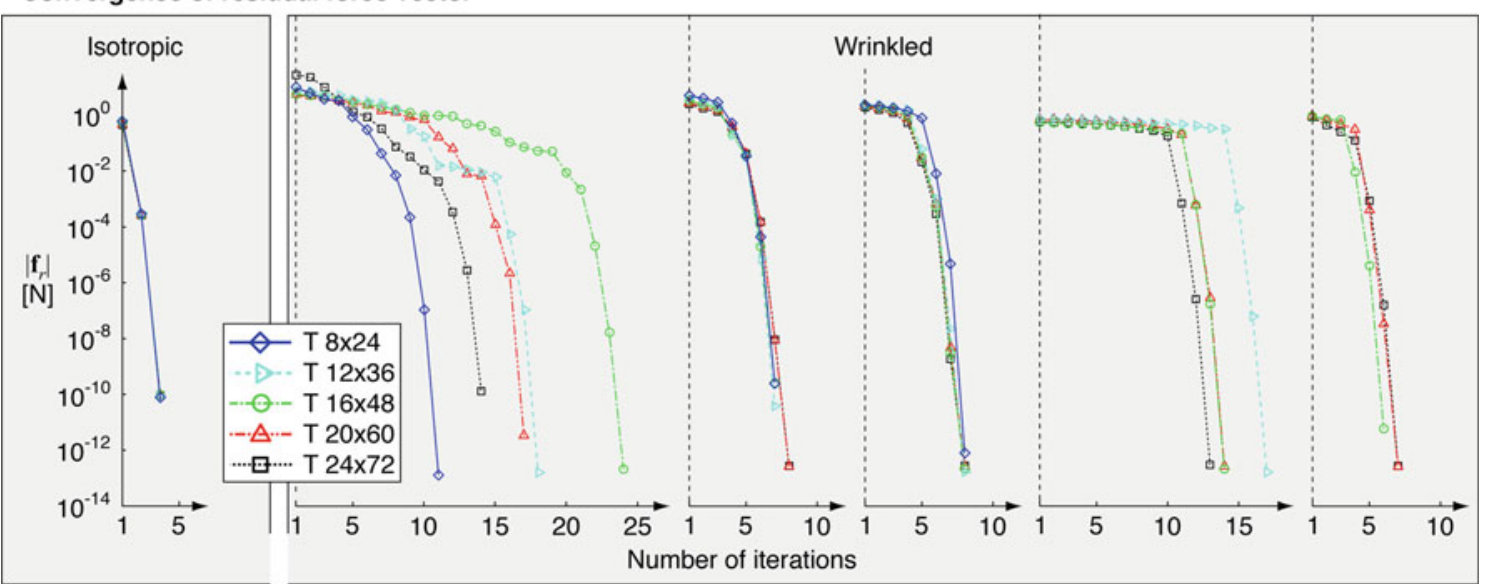

Fig. 10 Convergence of residual nodal force vector for different triangular and increasingly refined quadrilateral meshes. Applied displacements are $\Delta u=3 \mathrm{~mm}$ and $\Delta v=0$

and the vector a of size $e_{q}$ contains the known rotations $\alpha$ of the triangular elements

$\mathbf{a}=\left[\begin{array}{llll}\alpha_{1} & \alpha_{2} & \alpha_{3} & \cdots\end{array}\right]^{\mathrm{T}}$.

The least square approximation of the element rotations requires that $\mathbf{t}$ satisfies

$\left(\mathbf{a}^{\mathrm{T}}-\mathbf{t}^{\mathrm{T}} \mathbf{N}^{\mathrm{T}}\right)(\mathbf{a}-\mathbf{N} \mathbf{t})=\min$.

so that

$\mathbf{N}^{\mathrm{T}} \mathbf{N t}=\mathbf{N}^{\mathrm{T}} \mathbf{a}$.

Figure 9 shows the least square interpolation of the first principal stress directions of an unwrinkled isotropic membrane with three quadrilaterals. Since it is known that the tension field of a wrinkled membrane at an unstressed free edge has to be parallel to the latter, it is possible to enforce this boundary condition by fixing the rotations of the quadrilaterals at the corresponding nodes, Fig. 9. Another potential boundary condition is the continuity of the tension field and the first principal stresses between wrinkled and taut regions. The starting point for the following simulations is the interpolated tension field that enforces the boundary conditions at the free edges.
The stiffness matrix $\mathbf{K}=\mathbf{K}_{m}+\mathbf{K}_{g}$ and the force vector $\mathbf{f}$ that were derived in Sect. 3 are modified in the following to replace the element wrinkle direction $\alpha$ with the nodal rotations $\Theta$ of the surrounding quadrilateral. The multigrid force vector $\mathbf{f}^{\star}$ becomes

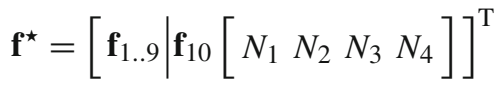

where $\mathbf{f}_{1 . .9}$ are the first nine entries of the force vector $\mathbf{f}$. The corresponding stiffness matrix $\mathbf{K}^{\star}$ results in

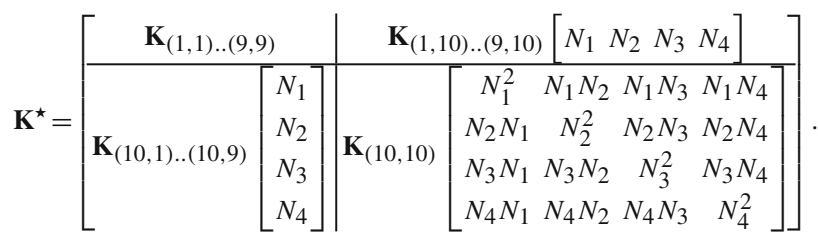

\section{Numerical examples}

This section demonstrates the good convergence properties of the multigrid approach by simulating a sheared $(\Delta v=0)$ rectangular membrane. A consequence of the pure shear is 

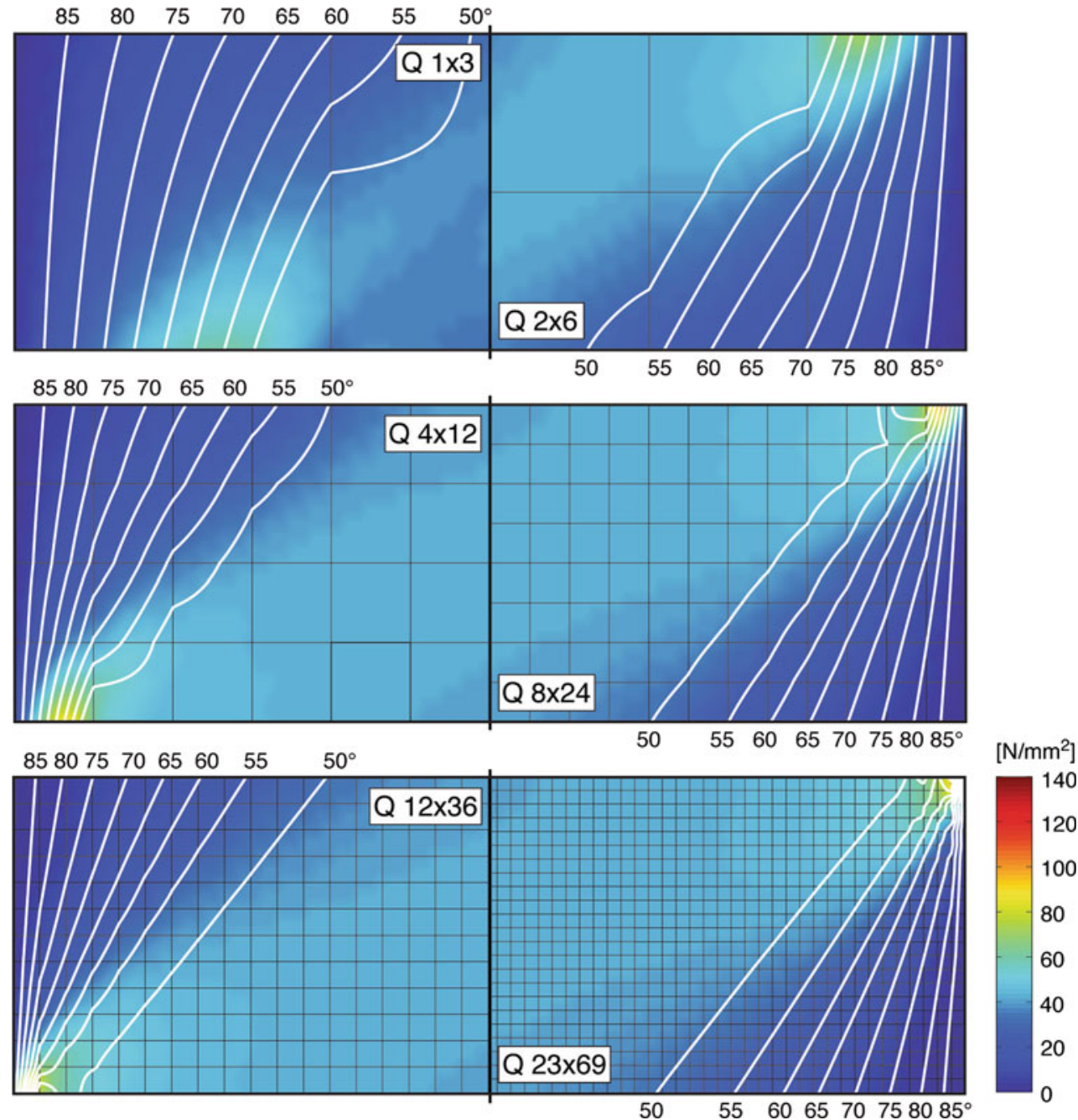

Fig. 11 Tension field for increasingly refined quadrilateral meshes (triangular background mesh is $\mathrm{T} 24 \times 72$ ). Applied displacements are $\Delta u=3 \mathrm{~mm}$ and $\Delta v=0$

the presence of two kind of singularities. The first one are infinite stresses and the second one are infinite gradients of the wrinkle directions at the bottom-left and top-right corners. Besides these singularities, there are regions around the topleft and bottom-right corners where the tension field stresses are very small. Therefore it is not surprising that existing numerical tools have difficulties to converge towards a continuous tension field throughout the membrane.

The nodal residual force vector from a previous simulation is unchanged if the tension field is interpolated with a refined quadrilateral mesh. However, the moments at the quadrilateral nodes are generally different after a mesh refinement. Since the used convergence criterion is based on the absolute value of the nodal residual force vector, it is necessary to compute the first iteration with a damped Newton-Raphson method ( $15 \%$ of step length). Subsequent iterations then use a line search algorithm that minimizes the absolute value of the nodal residual force vector.
Figure 10 shows the convergence plots of the residual nodal force vector for both, isotropic and tension field simulations on the basis of different triangular meshes T $m \times n$. The starting point for the tension field simulation is a quadrilateral mesh Q $1 \times 3$ that interpolates the directions of the first principal stresses from an unwrinkled isotropic membrane and enforces the boundary conditions at the free edges. This mesh is successively refined where the tension field from the previous step is used to initialize the subsequent simulation. It can be seen that the proposed method converges nicely to the desired equilibrium configurations within a few iterations for each quadrilateral mesh.

Figure 11 shows the tension rays for different angles and quadrilateral grids on the basis of a triangular background mesh of $\mathrm{T} 24 \times 72$. Note that the solutions of successively refined quadrilateral meshes converge towards the singularity at the bottom-left and top-right corners where the gradient of the wrinkle directions is infinite. Furthermore, it becomes 
Fig. 12 Comparison of analytical and numerical wrinkle directions and stress contours for $\Delta u=1.5 \mathrm{~mm}, \Delta v=0$ and T $24 \times 72$, Q $23 \times 69$

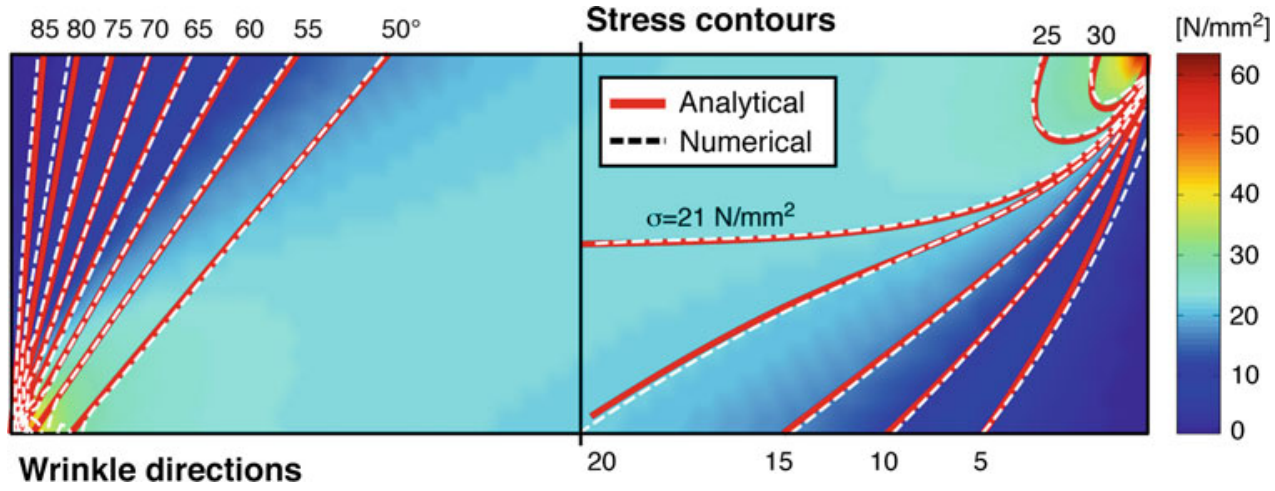

apparent that relatively coarse quadrilateral meshes like the Q $12 \times 36$ can accurately represent the tension field.

Figure 12 shows a comparison between the analytical and numerical solution for $\Delta u=1.5 \mathrm{~mm}$. The horizontal displacement was reduced to minimize geometric effects since the analytical solution is based on infinitesimally small displacements. ${ }^{2}$ The geometric effects are reflected by the slightly different values for the central stress contours. The central stress from the numerical solution is $21.12 \mathrm{~N} / \mathrm{mm}^{2}$ whereas the corresponding analytical stress is $21.01 \mathrm{~N} / \mathrm{mm}^{2}$. All other stress contours are plotted for the given values. The differences at the free edges are due to the finite triangular and quadrilateral mesh densities that can not represent an infinite gradient of wrinkle directions at the bottom left and top right corners. It can be seen that the stress contours from the numerical and analytical solutions are in good agreement. Furthermore, the tension field from the numerical solution is continuous and thus avoids any local optima.

\section{Conclusions}

This paper introduced a novel multigrid approach for the geometric non-linear simulation of highly wrinkled and stretched membranes on the basis of a three-node membrane finite element. The element possesses, in addition to the nodal displacement dof, an in-plane rotational dof inside the element domain that controls the direction of the tension field. This additional dof allows the enforcement of continuity and tension field boundary conditions on the basis of a coarser mesh with varying size. It was shown that the separate interpolation of displacements and rotations results in a good convergence behavior even in the presence of singularities that can exist in wrinkled membrane.

\footnotetext{
2 Steigmann [22] showed that small strain theories correspond to a singular limit of the general tension field theory, at which the underlying system changes from elliptic to parabolic type.
}

Acknowledgments This work was partially financed by St. John's College, Cambridge.

Open Access This article is distributed under the terms of the Creative Commons Attribution Noncommercial License which permits any noncommercial use, distribution, and reproduction in any medium, provided the original author(s) and source are credited.

\section{References}

1. Adler AL, Mikulas MM, Hedgepeth JM (2000) Static and dynamic analysis of partially wrinkled membrane structures. In: 41st AIAA SDM Conference

2. Akita T, Nakashino K, Natori MC, Park KC (2007) A simple computer implementation of membrane wrinkle behaviour via a projection technique. Int J Numer Methods Eng 71:1231-1259

3. Jarasjarungkiat A, Wüchner R, Bletzinger KU (2009) Efficient sub-grid scale modeling of membrane wrinkling by a projection method. Comput Methods Appl Mech Eng 198:1097-1116

4. Jenkins CH (2006) Recent advances in Gossamer spacecraft, volume 212 of progress in astronautics and aeronautics. AIAA

5. Kang S, Im S (1997) Finite element analysis of wrinkling membranes. J Appl Mech 64:263-269

6. Li X, Steigmann DJ (1993) Finite plane twist of an annular membrane. Q J Mech Appl Math 46(4):601-625

7. Liu X, Jenkins CH, Schur W (2001) Large deflection analysis of pneumatic envelopes using a penalty parameter modified material model. Finite Elem Anal Design 37:233-251

8. Lu K, Accorsi M, Leonard J (2001) Finite element analysis of membrane wrinkling. Int J Numer Methods Eng 50:1017-1038

9. Mansfield EH (1970) Load transfer via a wrinkled membrane. Proc R Soc A 316:269-289

10. Meyer M, Desprun M, Schröder M, Barr AH (2002) Discrete differential-geometry operators for triangulated 2-manifolds. In: VisMath

11. Mikulas MM (1964) Behavior of a flat stretched membrane wrinkled by the rotation of an attached hub. NASA technical note TN D-2456, NASA

12. Miller RK (1982) An algorithm for finite element analysis of partly wrinkled membranes. AIAA J 20(12):1761-1763

13. Mosler J (2008) A novel variational algorithmic formulation for wrinkling at finite strains based on energy minimization: Application to mesh adaption. Comput Methods Appl Mech Eng 197:1131-1146

14. Muttin F (1996) A finite element for wrinkled curved elastic membranes, and its application to sails. Commun Numer Methods Eng 12:775-785

15. Pagitz M, Pellegrino S (2009) Maximally stable lobed balloons. Int J Solids Struct 
16. Raible T, Tegeler S, Löhnert S, Wriggers P (2005) Development of a wrinkling algorithm for orthotropic membrane materials. Comput Methods Appl Mech Eng 194:2550-2568

17. Reissner E (1938) On tension field theory. In: Proceedings of the 5th international congress for applied mechanics, pp 88-92

18. Roddeman DG, Drukker J, Oomens CWJ, Janssen JD (1987) The wrinkling of thin membranes: Part 1 - theory. J Appl Mech 54:884887

19. Roddeman DG, Drukker J, Oomens CWJ, Janssen JD (1987) The wrinkling of thin membranes: Part 2 - numerical analysis. J Appl Mech 54:888-892

20. Rossi R, Lazzari M, Vitaliani R, Onate E (2005) Simulation of light-weight membrane structures by wrinkling model. Int J Numer Methods Eng 62:2127-2153
21. Schoop H, Taenzer L, Hornig J (2002) Wrinkling of nonlinear membranes. Comput Mech 29:68-74

22. Steigmann DJ (1990) Tension-field theory. Proc R Soc A 429: 141-173

23. Stein M, Hedgepeth JM (1961) Analysis of partly wrinkled membranes. Technical Report TN D-813, NASA

24. Tabarrok B, Qin Z (1992) Nonlinear analysis of tension structures. Computers and Structures 45(5-6):973-984

25. Wagner H (1929) Ebene blechwandträger mit sehr dünnem stegblech. Zeitschrift für Flugtechnik und Motorluftschiffahrt 20: 200-207

26. Wu CH, Canfield TR (1981) Wrinkling in finite plane-stress theory. Q Appl Math 39:179-199 